\title{
Las academias literarias áureas en torno a la narrativa corta de Alonso Jerónimo de Salas Barbadillo
}

\section{The Literary Academies of the Spanish Golden Age in the Short Stories of Alonso Jerónimo de Salas Barbadillo}

\section{Laura Yadira Munguía Ochoa}

Universidad Panamericana

Campus Guadalajara. Departamento de Humanidades

MÉXICO

Imunguia@up.edu.mx

[Hipogrifo, (issn: 2328-1308), 6.1, 2018, pp. 117-128]

Recibido: 11-01-2017 / Aceptado: 10-02-2017

DOI: http://dx.doi.org/10.13035/H.2018.06.01.09

Resumen. La proliferación de academias literarias del siglo XVII en el marco del Siglo de Oro español fue un espacio de comunicación y controversias entre escritores. La importancia de estas asociaciones es capital, ya que al interior de las tertulias surgen obras, alianzas y conflictos entre sus miembros; problemáticas que trascienden de lo personal a lo literario. La forma de trabajo y convivencia de las academias, tanto madrileñas como nacidas en provincia, se ven reflejadas dentro de la narrativa áurea. Un ejemplo de la ficcionalización de lo sucedido dentro de las instituciones intelectuales son las novelas cortas de Alonso Jerónimo de Salas Barbadillo, quien describe, con la narración acerca de academias ficticias, las problemáticas suscitadas en las reales.

El trabajo presenta un análisis histórico y temático de las obras en las que Salas Barbadillo habla de las academias, identificando las características y sucesos acaecidos en estas reuniones de escritores que sirven de sustento para sus novelas. Asimismo, se realizó una valoración de la narrativa de Barbadillo como una inspiración para la formación de academias, tanto del Siglo de Oro como en siglos posteriores, tomando en cuenta como punto esencial su organización, temáticas y formato. 
Palabras clave. Siglo de Oro; novela corta; academias literarias; Jerónimo de Salas Barbadillo.

Abstract. The proliferation of literary academies of the XVIIth century into the framework of the Spanish Golden Age generated a space of communication and controversies between writers. The importance of these associations is cardinal, because of all the alliances and conflicts that emerged between its members; problems that transcended from personal views to literacy. The dynamics and coexistence of the academies, both from Madrid and other provinces, are reflected on the Spanish Golden Age Narrative. The short novels of Alonso Jerónimo de Salas Barbadillo, are good example of the narrative in which Salas writes into fiction what took place within the academies.

This work presents a historical and thematic analysis of the narrations where Salas Barbadillo speaks about the academies, identifying the characteristics and events that took place among those writers' meetings that served as material for his novels. Likewise, Barbadillo's work was evaluated as a source of inspiration for further academy offspring, both during the Spanish Golden Age period and beyond, taking into account as essential point its organization, themes and format.

Keywords. Golden Age; Short Novel; Literary Academies; Jerónimo de Salas Barbadillo.

Las academias literarias jugaron un papel importante en el Siglo de Oro español, dando un espacio a los autores para compartir ideas, obras en proceso y mantener visibilidad en el mundo literario, en reacción a las problemáticas editoriales ${ }^{\top}$. Aunque hubo muchas academias áureas, de pocas tenemos conocimiento de sus actividades. La información nos viene de sus publicaciones, de los vejámenes y de las narraciones que tienen como escenario una academia. El presente trabajo rescata algunas descripciones de las academias literarias en la obra de Alonso Jerónimo de Salas Barbadillo, quien participaba activamente en las mencionadas instituciones. A través de un análisis histórico y temático de las obras de Salas, podemos observar las características y sucesos comunes dentro de las academias y su impacto en la literatura posterior.

Salas Barbadillo logra reflejar en su narrativa escenas reales acaecidas en las reuniones, haciendo una ficción que sirve tanto como un conocimiento de las academias, como una idealización del compartir literario y artístico. Aunque la aportación de Salas no es original ${ }^{2}$ en sus temas, sí es uno de los pioneros en usar

\footnotetext{
1. Milagros Rodríguez Cáceres en su artículo «Las academias como fiesta social del Barroco», expone acerca de las dificultades que los autores áureos enfrentaban para ver sus obras publicadas, hecho por el cual recurrían a otras opciones, la mejor de todas, las academias y las publicaciones derivadas de ellas, p. 108.

El pertenecer a una academia facilitaba las opciones de publicación, como lo demuestran las publicaciones basadas en las sesiones académicas. Ver Osuna, 2004.

2. Salas es uno de los primeros en usar el tema de la academia para publicar misceláneas, pero muy pronto otros autores seguirán la misma ruta: Arcadia de Lope de Vega; Cigarrales de Toledo y Deleitar aprovechando, de Tirso de Molina; Donaires del Parnaso de Alonso del Castillo Solórzano; La Cintia
} 
novelas misceláneas y con marco para describir academias. Unido a lo anterior, esta estrategia novelística era útil para incluir obras sueltas, que difícilmente podían publicarse de otra forma ${ }^{3}$.

Actualmente el interés por el madrileño ha tomado nuevo auge, no solo por sus obras capitales, como lo sería la Hija de Celestina, Don Diego de noche o la Estafeta del Dios Momo, sino también por sus novelas cortas, intercaladas dentro de sus narrativas misceláneas. Dentro de los estudios realizados tanto al autor como a su obra debemos destacar los trabajos de Émile Arnaud, Emilio Cotarelo y Mori, Edwin B. Place, Marc Vitse, Enrique García Santo-Tomás, Armine Manukyan, entre varios otros que se han dedicado en el siglo XX y lo que llevamos en el XXI del rescate y estudio de Salas Barbadillo4.

A Jerónimo de Salas (1581-1635) ${ }^{5}$ le tocó la buena suerte de nacer en uno de los momentos más gloriosos de la literatura española, siendo contemporáneo y formando parte de la estirpe más alta del Siglo de Oro español, pero esa misma suerte se tornó en mala fortuna, pues en medio de estos grandes autores su fama y trascendencia se vio opacada. Esta falta de popularidad en su época la podemos constatar en la considerable cantidad de dedicatorias que hace el autor a personajes de la nobleza y poemas laudatorios a los escritores más prestigiados en ese momento. Salas buscaba aceptación literaria y protección económica, recordemos que su familia se vio en dificultades pecuniarias, precisamente por el poco cuidado que tuvo de la herencia que por mayorazgo le pertenecía. Aunque no gozó de una fama comparable a la de Lope o Quevedo, Salas fue bien aceptado por sus contemporáneos y tuvo un éxito moderado con sus novelas, lo que le valió para lograr los elogios de Cervantes y el mismo Fénix de los ingenios, participando junto con ellos en academias y tertulias. Esta cercanía amistosa le sirvió también para el intercambio literario e influencias evidentes, sobre todo la de Cervantes, reflejadas en su obra6.

Varias de las circunstancias de su vida son determinantes para el estilo y proliferación de sus obras. En paralelo con la información que poseemos de otros auto-

de Aranjuez de Gabriel del Corral; Academias del jardín, de Polo de Medina; Academias morales de las musas, de Antonio Enríquez Gómez; Auroras de Diana de Pedro de Castro y Anaya; El Prado de Valencia, de Gaspar Mercader; La huerta de Valencia, de Alonso Castillo Solórzano, entre otras.

3. Comenta Milagros Rodríguez que tanto las misceláneas como las obras pastoriles son «excusa para la publicación de una nutrida antología poética», Rodríguez Cáceres, 2013, p. 107.

4. Destacamos las siguientes obras de los autores mencionados: de Émile Arnaud, Alonso Jerónimo de Salas Barbadillo: epitafios y seguidillas; de Emilio Cotarelo y Mori, Obras de Alonso Jerónimo de Salas Barbadillo; de Edwin B. Place (ed.), La casa del placer honesto de Alonso Jerónimo de Salas Barbadillo [1620]; de Marc Vitse, Salas Barbadillo y Góngora: burla e ideario de la Castilla de Felipe III; de Enrique García Santo-Tomás, Modernidad bajo sospecha. Salas Barbadillo y la cultura material del siglo XVII; de Armine Manukyan, «El necio bien afortunado de Salas Barbadillo. Rasgos genéricos».

5. Para ver detalles de la vida de Salas Barbadillo, ir a las ediciones de las obras hechas por Emilio Cotarelo y Mori (1907) y el libro de García Santo-Tomás (2008) quien nos da una extensa y detallada noticia de la vida de Salas.

6. Manukyan, 2012. Ahí encontramos un recuento de las obras donde existen elogios y reconocimientos entre escritores, tomando como ejes a Cervantes, Lope y Quevedo. 
res áureos, los datos de vida que conservamos de Barbadillo son reducidos. Émile Arnaud, Edwin Place y García Santo-Tomás, entre otros, logran dibujarnos a pesar de la poca evidencia documental, una vida de dificultades, de lucha por la pervivencia literaria, de limitaciones económicas y pérdidas familiares, pero también una existencia matizada con la aventura y las noches bohemias. La biografía de Barbadillo, en la que no podemos llevar un orden preciso y un por qué de varios acontecimientos, parece extenderse a las características generales de su obra, también coloreada por la diversidad de tonos y la concatenación de formas y espacios. Según Armine Manukyan: «Sus escritos son de variado contenido, extensión y forma que no se estriñen a un género determinado. Conviven en ellos distintas fórmulas narrativas y tonos posibles». Autor multifacético cuyas obras «son una especie de mezcla de elementos picaresco-costumbristas y satírico-burlescos»?.

Esta aparente diversidad sin norma ni ilación, donde se combinan estilos y géneros literarios, se mezclan tramas de diversa índole y pareciera un conglomerado de obras sujetas a una estructura nueva, es la principal característica de la obra de Salas y lo que la hace original e interesante ${ }^{8}$. El madrileño se debate entre dos estilos principales: el costumbrismo y la picaresca, aunque no se reduce a estos dos, sus novelas cortas de muy variado tono le hacen un autor multifacético.

Un valor acentuado de su obra se encuentra en sus novelas, varias de ellas caracterizadas por la metaficción, valiéndose de las historias con marco, populares en aquellos tiempos. La influencia de este estilo literario le venía por el lado italiano, considerando las coincidencias que tiene con el Decamerón, aunque no hay que descartar las influencias españolas. Dichas estructuras narrativas son fórmulas recurrentes en la obra de Barbadillo, caracterizándola de forma evidente. La novela corta intercalada en las historias con marco nos lleva a una riqueza de diversa índole, por un lado, la continuación tradicional de este tipo de estructuras, aunque con avances en la combinación de géneros como el costumbrismo y la picaresca o bien el tono serio y moralizante a un lado de la burla y la sátira. Plantea López Martínez: «Salas, pues, parece ser consciente de las dos variantes concretas que utiliza al momento de desarrollar sus propias obras, pero en ambos casos identificando siempre ese género narrativo con una utilidad moral»».

El autor nos muestra cómo se vale Salas con la estructura de sus novelas, para dotarnos de diversos temas e intenciones, características que se mantendrán a lo largo de su producción literaria. Inmerso en las modas literarias será partícipe de las novelas cortas en sus dos tipos, historias contadas dentro de otras historias y colecciones de novelas $\operatorname{cortas}^{10}$. Junto con Manuel Piqueras, podemos plantear que:

7. Manukyan, 2015, p. 185

8. Autores como Émile Arnaud (1981) han clasificado, en la medida de lo posible las obras de Salas, dividiéndolas en serias y de registro cómico.

9. López Martínez, 2014, p. 10.

10. No es la intención de este trabajo plantear las diferencias entre la colección de novelas cortas y las metaficciones con marco. Pero es importante señalar que la obra de Salas que comentaremos está inscrita en la segunda categoría señalada. 
En este sentido puede entenderse que todas las colecciones de novelas cortas que tengan un marco pueden considerarse también colecciones de metaficciones. Para ello solo es necesario que dentro de una ficción los personajes tengan conciencia de que las novelas que leen, relatan y escuchan son a su vez ficciones. Cabría distinguir entonces entre la ficción primaria (el marco) y la ficción secundaria (las novelas cortas insertas en él) ${ }^{11}$.

La casa del placer honesto ${ }^{12}$ de Salas, de acuerdo a los comentarios anteriores, sería una novela que introduce en metaficción, novelas cortas, a la par de otros géneros literarios. No estaríamos hablando entonces de una colección de novelas al estilo de Cervantes, sino más bien una historia con marco, que además, al introducir otros géneros se convertiría en miscelánea de complicada estructura. La mencionada novela de Salas, la cual se comentará en los siguientes párrafos, nos muestra de manera clara tanto la estructura miscelánea y de historia con marco, como la utilización del tema de las academias, no solo como un ejemplo de las tertulias, sino también como una estrategia literaria.

En 1620 se publica en Madrid la Casa del placer honesto, la historia principal presenta en forma de doble ficción varias novelas cortas, dentro de las cuales, observamos otros géneros como el teatro y la poesía insertados en las anteriores. La estructura general es muy cercana al Decamerón, aunque en opinión de Piqueras, nos encontramos con obras antagónicas, en el sentido del viaje de la ciudad al campo en el caso de la obra de Bocaccio y del campo a la ciudad, por el lado de Salas ${ }^{13}$.

Las academias fueron una institución importante en los siglos XVI y XVII, no tanto por su duración o los resultados de las mismas, sino por la congregación de grandes intelectuales y los avances en algunos casos en materia literaria, ejemplo de esto podría ser la importancia que llegó a tener la Academia de Madrid, en la que se citaban: Lope de Vega, Góngora, Quevedo, Tirso de Molina y el mismo Salas ${ }^{14}$. Aunque no haya una total certeza acerca de los integrantes de cada una de las academias, queda claro que varios de ellos participaron en más de una. Estas se volvieron un punto neurálgico áureo, «se convierte en un habitat en el que refugiarse, complementarse, reconocerse entre iguales», según palabras de Aurora Egido ${ }^{15}$. Funcionaron, sobre todo como una tertulia, pero también con el afán de lograr el favor de algún noble que se prestara como mecenas. Para Cañas Murillo «consistían tales Academias en reuniones de intelectuales que, eventualmente [...] o con cierta periodicidad y regularidad, se desarrollaban en determinadas casas de algunos nobles que se convertían en mecenas, en protectores, en patrocinadores de ellas» ${ }^{16}$.

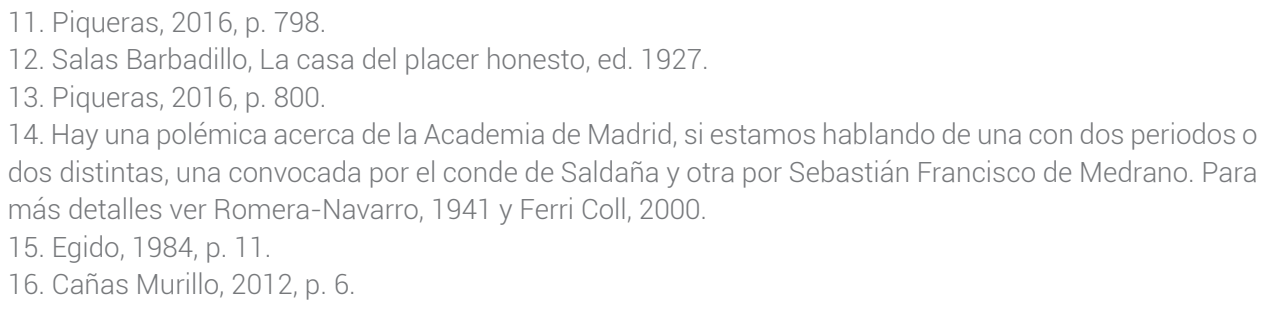


El primer problema era la periodicidad, sabemos que eran pocas aquellas que superaban los meses de vida, sin embargo su cantidad es considerable. Academias hubo que no fueron pensadas como una reunión periódica sino como celebración de un evento determinado, quedando extintas después de la celebración. Otras, más sólidas y conocidas, llegaron a mantenerse varios años, por ejemplo la academia valenciana de los Nocturnos ${ }^{17}$, donde se congregaron durante casi tres años personajes destacados y otros que tuvieron la fortuna de ser recordados en nuestros tiempos. Gracias a las actas y vejámenes ${ }^{18}$ conservados podemos darnos cuenta de varios detalles como su organización, miembros, fechas y horarios de reunión e incluso desavenencias, amistades y conflictos entre escritores. Los conflictos suscitados por lo general por las personalidades fuertes de los escritores, llegaron a terminarlas. De lo anterior nos queda constancia en varias fuentes, en primer lugar los vejámenes ${ }^{19}$, en segundo la poesía satírica y burlesca y en tercer ámbito, las narraciones que retratan los eventos comunes sucedidos. Para Ferri Coll «la habilidad que tiene el poeta de zafarse de la censura de la Academia y de su propia autocensura impuesta por sus mismos principios y de los imperantes en aquella sociedad, le da ocasión de exhibir su genio burlesco, aplicado a los asuntos más picantes, pero también a los más intrascendentes» ${ }^{20}$.

De acuerdo con lo mencionado por Ferri Coll, las disputas se centraban en la crítica entre académicos, no se discutían temas serios o delicados y los medios de crítica tenían que ver con sus disciplinas literarias, razón por la cual encontramos un sinnúmero de versos satíricos y burlescos, muchos de ellos sin duda nacidos al calor de las tertulias académicas. Sin embargo la fuente más rica, aparte de los vejámenes, para conocer lo sucedido en aquellas reuniones son las narraciones en las que se describe una academia ficticia.

Sabemos de varios autores que han dejado plasmado en novelas, dramas o fragmentos de las mismas, cómo eran las reuniones académicas del Siglo de Oro y qué solía suceder en ellas, como por ejemplo Lope de Vega, Castillo Solórzano entre otros, pero donde podemos ver un retrato más completo de una academia es

\footnotetext{
17. Sobre la academia de los Nocturnos nos quedan varios documentos que atestiguan sus reuniones, sus participantes y cómo se llevaba a cabo, además de una antología surgida de los trabajos en la institución. Para más información al respecto se recomienda revisar los trabajos de Sánchez, 1961; Mas i Usó, 1994 y 1999; y Cañas Murillo, 2012, entre otros.

18. Cañas Murillo, 2012, pp. 10-11. Cañas Murillo (2012) nos aclara cuál es la diferencia entre acta y vejamen, en la primera se trata de un documento donde se da cuenta de las actividades que se llevaron a cabo en las asambleas y por otro lado, un vejamen sería una narración de los hechos ocurridos durante las sesiones, la desventaja de este último es que al contar con lujo de detalles lo ocurrido durante las reuniones daba pie a incomodidades, enojos y susceptibilidades.

19. El término vejamen también nos lleva a textos satíricos y burlescos usados, no solo en las academias sino también en las universidades en el marco de los exámenes de grado (Madroñal Durán, 1994). Nos menciona también Rodríguez Sánchez de León (1989, p. 919) que «el juego literario de las academias no se daba por concluido sin antes amenizar a los asistentes con burla y el escarnio de los poetas concurrentes en el siempre esperado vejamen».

20. Ferri Coll, 2000, p. 328.
} 
en las novelas de Salas ${ }^{21}$. El madrileño incluye este tema en La peregrinación sabia, fabula en prosa, como él mismo la califica, incluida en Coronas del Parnaso y Platos de las musas ${ }^{22}$. También podemos ver aspectos de las tertulias literarias en parte de la trama del El sutil cordobés Pedro de Urdemalas ${ }^{23}$ y sobre todo en La casa del placer honesto ${ }^{24}$, en la que encontramos características muy puntuales de lo debieron de ser este tipo de instituciones del siglo XVII. Por medio de la creación de academias ficticias, Salas logra legarnos detalles que no hubiesen pervivido hasta nuestra época. El tema se vuelve un lugar común dentro de su obra, característica que obedece a la circunstancia social y literaria del momento ${ }^{25}$.

En La peregrinación sabia en forma de fábula, nos muestra las problemáticas dadas entre académicos, e incluso identifica con figuras de animales a varios autores famosos, o por lo menos se ha tratado de identificar a estos autores dentro de la narración. Para Romera-Navarro, el tordo se identifica con Pedro Torres Rámila, el ruiseñor, con Lope de Vega, el perro con Cristóbal Suárez de Figueroa y el gato con Luis Vélez de Guevara ${ }^{26 .}$

El episodio de la academia se encuentra como una de las aventuras que pasan dos zorros, padre e hijo, en su recorrido por el mundo, con la intención de que el joven zorro aprendiera de la naturaleza humana y tomara experiencia, tanto del mundo como del padre. Después de varias tretas de las cuales los zorros, debido a su astucia y retórica, salen triunfantes, se detienen a descansar y refrescarse. Es en este lugar donde presencian una sesión de la academia. En El sutil cordobés Pedro de Urdemalas, una novela que no tiene como eje este asunto, se nos muestra la dinámica común llevada a cabo en las tertulias.

La casa del placer honesto trata de la reunión voluntaria de cuatro jóvenes de familias poderosas, junto con sus sirvientes, con el fin de compartir el arte y las finas formas de manera exquisita. Cabe destacar que los jóvenes se encargaban exclusivamente de tareas intelectuales y refinadas, dejando todo lo mundano a sus ayos y la administración de la casa en manos de un mayordomo. El dinero es provisto por sus padres como parte de su herencia y no tiene mayor significado que aquel que les facilita una vida cómoda y con lujos, en un ambiente puramente burgués y cortesano. Los jóvenes artistas no se preocupan por el futuro, sino por los placeres refinados que pueden disfrutar en el presente.

El proyecto de academia inicia con la protesta de don Diego, quien se queja de las pocas oportunidades de conocimiento y placer que les permite su residencia en Tormes, además de lo limitada que resulta la vida universitaria. Por lo anterior conmina a sus amigos: don García, don Fernando y don Próspero para que, obligando a sus padres a su sostenimiento, se muden cerca de la corte:

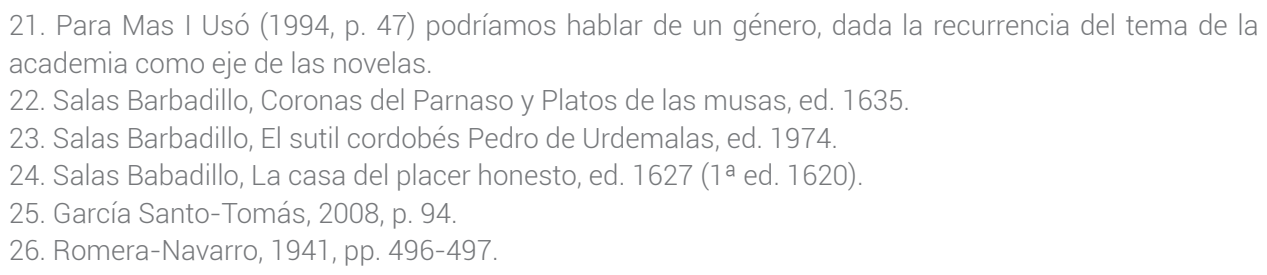


Vámonos a la corte vivamos allí con los alimentos y socorros que es fuerza que nos envíen nuestros padres; en apacible y no escandaloso deleite fundaremos una casa del placer honesto, cuyas constituciones determinaremos entre los cuatro, guardándolas inviolablemente a los que quisieren estar en nuestra compañía, que lo demás que hoy hacemos no es elección útil ni descansada27.

Deciden rentar un solar de amplias habitaciones y jardines, con el fin de usarla tanto de residencia como de espacio de reunión académica. Los cuatro jóvenes, acaudalados y sin compromisos inician sus actividades, nombrando su asociación como Casa del placer honesto, debido a que estará destinada a los placeres finos y virtuosos del arte y el pensamiento.

Para poner en marcha la academia, formulan una serie de estatutos, que consideran inviolables y necesarios para la buena convivencia y desarrollo artístico. Es interesante observar en su insistencia en el alejamiento de la universidad y fijar, como mejor opción cultural el autodidactismo. Las ordenanzas se distinguen por su severidad y elitismo. Los cuatro jóvenes se alejan del mundo como una forma de mejor cultivo de las artes, pero también desde un punto de vista excluyente, donde la muchedumbre, las tradiciones y los espectáculos públicos constituyen algo grosero, fútil y vulgar28.

El primer estatuto 29 propone algo radical, ninguno de los miembros puede ser casado, ni tener relación amorosa alguna, ya que de estas se desprenden pesares e inconveniencias que no suceden sino por gusto propio, lo cual no se considera prudente; con esto se sobreentiende el celibato. Llama la atención que en diversas ocasiones son cantados poemas amatorios, y mostradas relaciones amorosas en las novelas cortas; hecho que nos da a entender que los poemas no tienen un trasfondo, sino que se presentan como un ejercicio literario.

Los fundadores se comprometen a no asistir a evento alguno que tenga que ver con el vulgo o las masas, asimismo, se prohíbe la entrada a toda persona que no mantenga una visión esteticista, artística y en cierto modo hedonista, por la concepción que muestran sobre el placer. Se prohíbe también la entrada a personas que por su nivel socioeconómico muy alto o muy bajo, les pueda distorsionar la visión del placer del arte y el pensamiento. Los miembros de la academia deben estar alejados de toda reunión social, y ser eminentes exponentes de las artes poética, oratoria y musical como mínimo.

27. Salas Barbadillo, 1927, pp. 329-330. Se modernizan las grafías sin relevancia fonética siguiendo las normas de Hipogrifo. El episodio es citado también por Piqueras, 2016, p. 800.

28. Dentro de la revisión histórica que hace Egido, observamos que la academia planteada en La casa del placer honesto se parece más a las surgidas en el Renacimiento italiano, de tintes filosóficos, herederas aún de la platónica, que las que se desarrollaban en el Siglo de Oro, en las que «de la discusión filosófica se pasó al debate pueril y al verso ligero». Las academias italianas, se alejan de los fines prácticos, se aíslan y tratan de seguir los ideales grecolatinos, como vemos que sucede en la obra de Salas Barbadillo (Egido, 1984, pp. 10-11).

29. Podemos ver la descripción de los estatutos en Salas Barbadillo, La casa del placer honesto, pp. 330-333. 
Sabemos que Salas Barbadillo era afecto a describir las academias a las que asistía, de forma parodiada y mostrando las situaciones que se suscitaban puertas adentro, característica que se hace común dentro de su obra ${ }^{30}$, sin embargo, el caso de La casa del placer honesto es especial, ya que nos muestra una idealización del funcionamiento de una reunión literaria. La forma de organización ${ }^{31}$ de esta tertulia trata de emular a las reales, aunque, por supuesto, con situaciones exageradas e idealizadas.

Salas propone una idea de convivencia académica y de funcionamiento de tertulia ideal y poco verosímil por la serie de normativas estrictas y muy difícil de cumplir en el plano real. La idea de academia mostrada por Salas es un espacio sublimado, que más se parece a un ambiente conventual que al de una academia común del siglo XVII. Un tipo de vida dedicada al estudio, la creación y los placeres honestos, muy alejado de la mundanidad de los escritores áureos, los verdaderos académicos. Salas se inserta en una fórmula literaria usada por sus contemporáneos, donde podían mezclar géneros y mostrar algunas de las vicisitudes suscitadas en la reuniones académicas, pero esta nueva representación planteada por el madrileño establece un ideal artístico ajeno a disputas, en el que lo importante es el disfrute artístico y no la lucha por el reconocimiento público y la obtención del apoyo de un mecenas. Desde este punto de vista la obra de Salas será una valiosa pieza en la formulación posterior de este tipo de reuniones tanto ficticias como reales.

La novela se desarrolla en un ambiente cerrado y reducido, en el cual pueden pasar diversas situaciones, "honestas", como en este caso sucede. Los personajes de Salas son nobles, de formas y gustos refinados, pertenecientes a familias acaudaladas que habían facilitado a los jóvenes una esmerada educación, muy diferentes de la realidad literaria del siglo XVII, matizada por una variedad de autores pertenecientes a todas las condiciones y medios socioeconómicos. Recordemos para esto la comparación que hace Rey Hazas entre las diferentes personalidades de Cervantes y Quevedo, ambos, copartícipes de las academias a las que asistía Salas Barbadillo: «Entre nuestros dos escritores, en cualquier caso, hubo sobre todo diferencias de toda índole: diferencias de tiempo, de talante, de intereses, de personalidad y de estética»32.

La dinámica que muestra Salas en su novela es muy esquemática; se conviene en un día y hora específico para la lectura, canto o interpretación de las obras creadas por los miembros de la academia. En ocasiones se designa el género a cumplir

30. Salas Barbadillo, Coronas del Parnaso y Platos de las Musas, pp. 81-85. Esto podemos observarlo sobre todo en el episodio de la academia dentro de La peregrinación sabia, lo que se nos hace evidente no es una idealización de las academias, sino los problemas, envidias y traiciones que se suscitan dentro de las reuniones.

31. En el comentario que hace Rodríguez Sánchez de León a propósito del artículo de Carrasco Urgoiti, nos dice que en una academia «la velada tenía su inicio cuando el presidente pronunciaba la oración inaugural que, a su vez, daba inicio a la intervención del secretario. A continuación se exponían los asuntos sobre los que se habían de versar las composiciones de los poetas concurrentes y se concluía con el vejamen de los resultados obtenidos» (Rodríguez Sánchez de León, 1989, p. 916).

32. Rey Hazas, 2008, p. 201. 
y otras veces se impone también el tema e incluso el título, como pasa en el caso de los dos jóvenes que piden su admisión en la casa: don Juan y don Alonso. Para su ingreso deben aprobar un examen, que consiste obviamente en la muestra de su talento poético y narrativo. Es curioso notar que una de las novelas cortas insertadas más largas y mejor trabajadas es la pronunciada por don Alonso, que nos recuerda al mismo Salas.

El desarrollo de las tertulias es lo que hace caminar la novela, marcada primero por el establecimiento de los estatutos, y después por la presentación intercalada de relatos y poemas pronunciados por los miembros, aparentemente desvinculados de la historia general del marco. La trama carece de acción y sostiene la misma dinámica: la academia se reúne y cada uno narra o recita su trabajo, al final todos aplauden y celebran el ingenio de su compañero.

La novela tendría dos puntos de interés esenciales, uno referente a la historia del marco, es decir, la presentación de la academia y sus características, por otro lado, las temáticas de las novelas cortas narradas por los miembros de la tertulia; relatos donde se concentran los elementos lúdicos, satíricos y picantes, sin contar el manejo fluido de la acción de los personajes. El conjunto narrativo que nos ofrece Salas en esta novela se presenta en varios niveles del relato, el primero es la historia con marco, donde uno de los personajes cuenta una historia; la estructura se complica cuando en la novela corta: El coche mendigón, envergonzante, y endemoniado, narrada por don Alonso, es relatada en varios planos, es decir una historia dentro de otra, hasta llegar a la central de Cristina y su obsesión por los coches, lo que ya en sí constituye una idea interesante y lúdica. Cada una de estas breves novelas tiene su interés por separado.

Este empalme complicado de tiempos, espacios y personajes nos deja ver o bien una estrategia rebuscada para narrar historias, o la creación de un modelo de novela marco que ajustara a las historias sueltas, sirviendo como un recipiente de unidad y cohesión. Según García Santo-Tomás, «son piezas de muy distinto contenido que nuestro autor debió tener guardadas durante años previos y que, aprovechando la oportunidad de publicar, insertó en el texto principal, ocupando la gran mayoría de su volumen» ${ }^{33}$. Ambas formas de acercamiento nos muestran a un autor valioso y experimentado que sabía usar a su favor los recursos literarios en boga, como la moda de las novelas misceláneas y con marco, con el fin de publicar fragmentos sueltos o colecciones de poemas y narraciones.

Salas Barbadillo logra conformar una valiosa obra, profusa en ingenio y elocuencia. El autor, logró también escribir en varios géneros de manera destacada, pero sobre todo supo cómo mezclar géneros y temas complicando sus argumentos y haciendo con sus tramas una literatura original y atrayente. A la par de sus estructuras, es de mencionar que Salas es uno de los autores que plasma en sus novelas la realidad de las academias literarias del siglo XVII, con sus características y peripecias, pero también reflejando los deseos intelectuales de su época. Vale la pena acercarse más a Salas Barbadillo, quien sin duda, tiene mucho más que decirnos.

33. García Santo-Tomás, 2008, p. 95. 


\section{BiBLIOGRAFÍA}

Arnaud, Émile, «Alonso Jerónimo de Salas Barbadillo: epitafios y seguidillas» Criticón, 14, 1981, pp. 5-42.

Cañas Murillo, Jesús, «Corte y academias literarias en la España de Felipe IV», Anuario de Estudios Filológicos, vol. XXXV, 2012, pp. 5-26.

Cotarelo y Mori, Emilio, Obras de Alonso Jerónimo de Salas Barbadillo, Madrid, Tipografía de la «Revista de Archivos», vol. I, 1907, vol. II, 1909.

Ferri Coll, José María, «Burlas y chanzas en las academias literarias del Siglo de Oro: Los Nocturnos de Valencia», en Actas del XIII Congreso de la Asociación Internacional de Hispanistas, Madrid 6-11 de julio de 1998, coord. por Florencio Sevilla Arroyo y Carlos Alvar Ezquerra, vol. 1, 2000, pp. 327-335.

Egido, Aurora, «Una introducción a la poesía y a las academias literarias del siglo XVII», Estudios humanísticos, 6, 1984, pp. 9-26.

García Santo-Tomás, Enrique, Modernidad bajo sospecha. Salas Barbadillo y la cultura material del siglo XVII, Madrid, Consejo Superior de Investigaciones Científicas, 2008.

Lacadena y Calero, Esther, «El discurso oral en las academias del Siglo de Oro», Criticón, 41, 1998, pp. 87-102.

López Martínez, José Enrique, «Corrección de vicios de Salas Barbadillo, y la primera etapa de la novela corta española», Lejana. Revista Crítica de Narrativa Breve, 7, 2014, pp. 1-16.

Madroñal Durán, Abraham, «Sobre el Vejamen de Grado en el Siglo de Oro. La Universidad de Toledo», Epos, Revista de Filología, 10, 1994, pp. 203-231.

Manukyan, Armine, «El necio bien afortunado de Salas Barbadillo. Rasgos genéricos», Foreing Languages in Higher Education, Universidad Estatal de Erevan, (Armenia), 18.1, 2015, pp. 185-191.

Manukyan, Armine, «Salas Barbadillo entre sus contemporáneos: sus gustos literarios e influencias», en Scripta manent. Actas del I Congreso Internacional Jóvenes Investigadores Siglo de Oro (JISO 2011), ed. Carlos Mata Induráin y Adrián J. Sáez, Pamplona. Servicio de Publicaciones de la Universidad de Navarra, 2012 (Publicaciones digitales GRISO), pp. 279-295.

Mas I Usó, Pasqual, «Academias ficticias valencianas durante el Barroco», Criticón, 61, 1994, pp. 47-56.

Mas I Usó, Pasqual, Academias valencianas del Barroco. Descripción y diccionario de poetas, Kassel. Edition Reichenberger, 1999.

Piqueras Flores, Manuel, «Alonso J. de Salas Barbadillo y las colecciones de metaficciones áureas», Castilla. Estudios de Literatura, 7, 2016, pp. 794-811. 
Osuna, Inmaculada, «Aproximación a las Academias granadinas del siglo XVII», en Memoria de la palabra. Actas del VI Congreso de la Asociación Internacional Siglo de Oro (AISO), ed. María Luisa Lobato y Francisco Domínguez Matito, vol. 2, Madrid, Iberoamericana/Vervuert, 2004, pp. 1401-1409.

Rey Hazas, Antonio, «Sobre Quevedo y Cervantes», La Perinola, 12, 2008, pp. 201229.

Rodríguez Cáceres, Milagros, «Las academias como fiesta social del Barroco: su reflejo en Antonio Enríquez Gómez», Hipogrifo. Revista de literatura y cultura del Siglo de Oro, 1.1, 2013, pp. 105-119.

Romera-Navarro, Miguel, «Querellas y rivalidades en las Academias del siglo XVII», Hispanic Review, 9.4, 1941, pp. 494-499.

Salas Barbadillo, Alonso Jerónimo, La casa del placer honesto de Alonso Jerónimo de Salas Barbadillo [1620], ed. Edwin B. Place, Colorado, University of Colorado Studies, vol. XV, 4, 1927

Salas Barbadillo, Alonso Jerónimo, Coronas del Parnaso y Platos de las Musas, Madrid, Imprenta del Reino, 1635.

Salas Barbadillo, Alonso Jerónimo, El subtil cordovés Pedro de Urdemalas y El gallardo Escarramán, ed. Marcel Charles Andrade, Madrid, Castalia, 1974.

Sánchez, Jesús, Academias literarias del Siglo de Oro español, Madrid, Gredos, 1961.

Rodríguez Sánchez de León, María José, «La academia literaria como fiesta barroca en tres ejemplos andaluces (1661, 1664 y 1672)», en El teatro español a fines del siglo XVII. Historia, cultura y teatro en la España de Carlos II, ed. Javier Huerta Calvo, Harm der Boer y Fermín Sierra Martínez, Diálogos Hispánicos de Amsterdam, 8, III, 1989, pp. 915-926.

Vitse, Marc, «Salas Barbadillo y Góngora: burla e ideario de la Castilla de Felipe IIl», Criticón, 11, 1980, pp. 5-42. 